\title{
Stabilization of Silicone Dioxide Nanoparticle Foam in Tertiary Petroleum Production
}

\author{
Mohd Zulkifli bin Mohamad Noor ${ }^{1, *}$, Wong Yi Teng ${ }^{1}$, and Sonny Irawan ${ }^{2}$ \\ ${ }^{1}$ Faculty of Chemical and Natural Resources Engineering, Universiti Malaysia Pahang, \\ Lebuhraya Tun Razak 26300, Gambang, Pahang, Malaysia \\ ${ }^{2}$ Department of Petroleum Engineering, Faculty of Engineering, Universiti Teknologi PETRONAS, \\ 32610 Seri Iskandar, Perak Darul Ridzuan, Malaysia
}

\section{* Corresponding author:}

tel: $+60-127399801$

email:mzulkifli@ump.edu.my

Received: August 3, 2018

Accepted: December 11, 2018

DOI: $10.22146 / \mathrm{ijc} .40870$

\begin{abstract}
Nanoparticles have emerged with substantially to the end user and industrial applications. The applications initiated to enhance oil recovery (EOR) and also as an alternative solution in increasing the rheological properties offluids at different condition. The study aims to evaluate the effects of various surfactant and nanoparticle concentration as well as hydrocarbons on foam stability. Series of static state experiments were conducted to investigate the foam development stability of five different concentrations for surfactant from 0.05 to $0.25 \mathrm{wt} \%$ and nanoparticle from 0 to 1.00 $w t . \%$ in the presence of white mineral oil in synthetic brine suspension. By discussing the Ross-Miles method - half-life capacities ( $\left.t_{1 / 2}\right)$, the foam stability of the aqueous foam was expected. Results suggested that the foam stability increased with the presence of a nanoparticle. The $0.5 \mathrm{wt}$ \% $\mathrm{SiO}_{2}$ nanoparticles enhanced foam formed the most lasting in the absence of white mineral oil as its $t_{1 / 2}$ in the presence of oil is 0.6 times smaller than in the absence of oil. It is concluded that the presence of nanoparticles for surfactant foam stability can be enhanced. The used of nanoparticles can be further study with a different type of nanoparticles, only with a small amount of nanoparticles used can further stabilize the foam.
\end{abstract}

Keywords: enhanced oil recovery; half-life measurements; mobility ratio; rheological

\section{- INTRODUCTION}

Ordinarily, EOR that is known as tertiary recovery incorporates the process that diminishes the residual oil saturation by gas and/or chemical injection, as well as thermal recovery methods [1]. However, the large mobility ratio between the displacing gas and oil phases is a critical challenge to encounter in the EOR process. Therefore, such low-viscosity gas is more volatile than displaced oil or water in the reservoir. Leading it to channel through the reservoir that resulted in the early breakthrough of gas with a large volume of hydrocarbon trapped in reservoir.

Moreover, the low-density gas fluid relative to oil will readily result in the gravity segregation, which inhibits the contact between the displacing gas and deeper hydrocarbon zones. The above problems of viscous fingering and gravity segregation are considered to be an unfavorable displacement condition due to a chance in occurrence whereby large oil quantities in the formation will be bypassed as well as the formation of residue oil saturation that proves to be at high in the case of gas breakthrough [1]. In order to achieve a lower mobility ratio of solvent to reservoir oil, it is necessary to find a mobility control method to make the displacement sustain a uniform displacement front.

Considering the fact that foams have been utilized in well-nigh every level of the petroleum production as well as a refining process, starting with fathomless producing reservoirs, following by oil well drilling, stimulation, and production, till downstream vessels, foam stability factor has been accentuated in the field involved [2]. According to Gonzenbach et al. [3] foam generated by a surfactant is prompt to be inadequate for mountainous scale operation like EOR as they are most 
inclined to deteriorate chemically in the beginning even before executing their purpose. The inability of this foam to maintain long-term stability occurs especially at elevated temperature or with the presence of oil or brine. In addition to the study by Grigg and Mikhalin [4], there are also chances for the surfactant to induce high surface retention condition as there is potential for it to be adsorbed into the rock. Isari et al. [5] said it also could be used using a natural surfactant to recover the interfacial tension (IFT) by crude oil. Particles materials respond similarly with surfactant in term of factors influencing the stability that are generally confided in concentration, shape, size of particle and their hydrophobicity [6].

The application of foam in tertiary oil recovery had been gaining popularity, and it is none other but due to its capability of decreasing the gas-phase mobility considering it is a mobility control agent. Another approach being done through clinical study for human vascular in vitro process for it foam stability in foam sclerotherapy in vitro [7]. Therefore, generating foam in situ can be a viable approach to mitigating the sweep inhomogeneity and increase the sweep efficiency of solvent flood due to the foaming mechanism. The high conspicuous viscosity of foam granted it low mobility of displacing fluid which is critical to improving the volumetric sweep efficiency of the solvent flood to improve the oil recovery. Conventional foam, generated in situ by surfactantalternating-solvent injection is extensively practiced in the oil and gas industry. Due to its amphiphilic property, a surfactant is considered as an effective foam forming agent. In another way of speaking, the hydrophilic head of surfactant has the affinity to the water phase, while its hydrophobic tail has the affinity to gas or oil [8].

With the growing interest among researchers in the time frame of these few years regarding the employment of nanoparticle materials, the replacement for the surfactant stability provide partial remedy to the limitation of the traditional surfactant-stabilized foam. However, early studies on this field had already been conducted back in the late '80s [9] and further brought forward in the study regarding food in 2004 [10]. Henceforth nanotechnology, through the mean of nanoparticles, has paved an alternative solution in heightening the fluids rheological properties at ambient and elated temperatures, even though, detrimental significant is significant at particular nanoparticle concentrations [11]. The stability of nanoparticles in solutions confide on the performance as well as its surface activity. Comprehensively, the surface of the nanoparticles is usually functionalized by laying shields encompassing them. The presence of these shields trammels any form of particle-particle interaction while reducing the feasibility of nanoparticles aggregation [10]. Therefore, the objectives of the present work are to study the effects of various surfactant and nanoparticle concentration as well as hydrocarbons with well-defined on its concentration and viscosity properties on foam stability.

\section{- EXPERIMENTAL SECTION}

\section{Materials}

Surfactant (sodium dodecyl sulfate) known volume was diluted in the deionized water. Taking for two hours shaken for the solution to obtain a homogenous solution using Orbital Shaker at $240 \mathrm{rpm}$. Then, nanoparticle (silicon dioxide) is added to the solution. Brine and white mineral oil are added if necessary. The dispersion solution was sonicated for four hours to obtain a miscible equilibrium. The magnetic stirring helps to disperse the nanopowders evenly in the base fluid, but the energy was not enough to break any agglomeration of nanoparticles. So an ultrasonic bath was used to break the agglomerations of nanoparticles. The nonionic surfactant was not sensitive to salinity variations because it largely relies on steric repulsive force to disperse the nanoparticle. This was desirable to select nonionic surfactants as good candidates, especially for high salinity conditions. The hydrophobic tail of the nonionic sodium dodecyl sulfate surfactant (SDS) is non-covalently adsorbed onto the silicon dioxide $\left(\mathrm{SiO}_{2}\right)$ nanoparticles surface [12], while the hydrophilic head provides strong steric repulsion to prevent aggregation and improve the dispersion of nanoparticles. 


\section{Instrumentation}

The FTIR spectra of $\mathrm{SiO}_{2}$ nanoparticles with SDS concentration of $0.25 \mathrm{wt} . \%$ and brine solution at $0,0.1$, $0.3,0.5$, and $1.0 \mathrm{wt}$ \% concentration, respectively from 400 to $4000 \mathrm{~cm}^{-1}$ were obtained using Perkin Elmer's ATRFTIR Spectrometer (Frontier). The spectra were analyzed using OMNIC software with an average scan of 16 .

\section{Procedure}

\section{Static bulk foam test}

Bulk foam experiments can be static or dynamic. The foam stability test was set and improved based on ASTM-D 6082-62 [13]. In this study, the static stability of the aqueous foam was expected by referring to the RossMiles method [14], using its half-life measurements. However, the half-life was determined by determining the life span essential by the foam to channel half of its initial volume. The approach practiced in a spread of foam is air expansion. This approach was used to appraise the stability of foam in the absence of nanoparticles, surfactant, brine and white mineral oil. The equipment used consisted of $1 \mathrm{~L}$ graduated cylinder, a gas flow meter, and a high purity carbon dioxide tank. The standard volume of ready solution was introduced into the graduated cylinder. At this point, carbon dioxide $\left(\mathrm{CO}_{2}\right)$ gas was allowed to flow through tube into the bottom of the solution. After a standard volume of $\mathrm{CO}_{2}$ (about $500 \mathrm{~mL} / \mathrm{min}$ ) was introduced, the flow meter was closed,

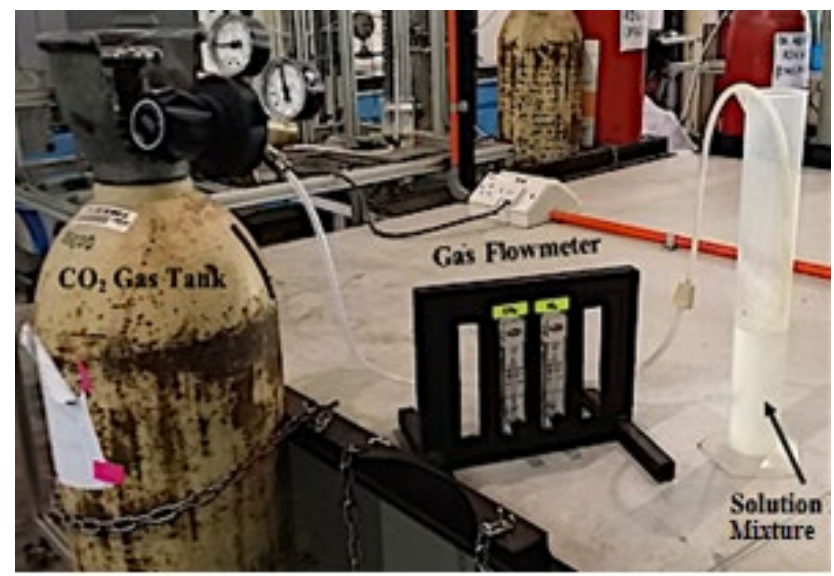

Fig 1. Experimental setup of the foam stability at static bulk scale with the gas flow controller for air flow control and the foam stability was determined by the foam layer thickness versus the time [14].

Additionally, as to characterize the foam stability, normalized foams height were measured and calculated by using Eq. 1 [15].

Normalized height $=\frac{\text { foam height }(t)}{\text { foam height }(t=0)}$

where $\mathrm{t}$ is time in $\min$.

\section{Half-life test}

Half-life tests for the foaming agents are conducted to decide the crucial foaming agent concentration to be mixed with water. Surfactant and nanoparticle were mixed with different concentrations in water (SDS: 0.05, $0.1,0.15,0.2$, and 0.25 wt.\%; Nanoparticles: 0, 0.1, 0.3, 0.5 , and $1.0 \mathrm{wt} . \%$ ) and agitated by means of an Orbital Shaker, and then moved into a graduated cylinder. Initial total foam volume is measured and documented. The volume of liquid phase collected at the bottom of the graduated cylinder is also detailed as a function of time. When half of the total liquid volume came out at the bottom of the graduated cylinder, the time of this period is noted as the half-life of the foam. This method [16] is based on the measurement of the liquid phase drained volume as a function of time. Foams mixture solution are to be prepared through stirring at $240 \mathrm{rpm}$ of $150 \mathrm{~mL}$ of the liquid phase in stirrer for $2 \mathrm{~h}$ and dispersed for $4 \mathrm{~h}$ in an ultrasonic bath. The prepared foam mixture solution is immediately transferred to a $1 \mathrm{~L}$ graduated cylinder and the drained volume of the foam in term of height in the graduated cylinder is recorded every $1 \mathrm{~min}$ for $10 \mathrm{~min}$. Besides this, as half of the total liquid phase has been taken a few time to be recorded as half-life for this surfactant/nanoparticle mixture. Below are shown three equivalent formulas describing exponential decay [16].

$$
\begin{aligned}
& \mathrm{H}(\mathrm{t})=\mathrm{H}_{\mathrm{o}}\left(\frac{1}{2}\right)^{\frac{\mathrm{t}}{\mathrm{t}_{1 / 2}}} \\
& \mathrm{H}(\mathrm{t})=\mathrm{H}_{\mathrm{o}} \mathrm{e}^{-\frac{\mathrm{t}}{\tau}} \\
& \mathrm{H}(\mathrm{t})=\mathrm{H}_{\mathrm{o}} \mathrm{e}^{-\lambda \mathrm{t}}
\end{aligned}
$$

where $H_{t}=$ remaining foam height after time, $t ; t_{1 / 2}=$ half time; $\tau=$ mean time; and $\lambda=$ decay constant. 


\section{- RESULTS AND DISCUSSION}

\section{Surfactant Screening}

The concentration of SDS surfactant is crucial to foam stability and, based on these results, whereby, stronger foam can be produced using $0.25 \mathrm{wt} . \%$ of SDS surfactant for both conditions. Optimum behaviors were observed at 0.25 wt.\% SDS surfactant. Fig. 2 shows that, based on relative foam height measurements (normalized height), 0.25 wt.\% SDS surfactant produces more stable foams at both absence and presence of white oil with normalized height of 0.51 and 0.42 respectively. The foam is found to be less stable in the presence of white oil as SDS solution mixture provide lower foam height at all 5 concentrations when compared to those in the absence of white oil. Fig. 2 showed drastic lower foam height for SDS in white oil with very weak stability in which foam level diminishes as gas injection ceases after $10 \mathrm{~min}$. As concentration increases above 0.02 wt. $\%$, a good foam height with reasonable stability and oil displacing efficiency was obtained.

In both cases, the concentration of 0.05 wt.\% of SDS surfactant produces the weakest foam with the lowest normalized height obtained from the experiment which is 0.37 in the absence of white oil and 0.33 in the presence of white oil. SDS foam was characterized by a fairly uniform and broad bubble size distribution. Soon after the end of gas sparging, the foam column entered the decay reign. The 0.25 wt. $\%$ of SDS foam formed the most stable in the absence of oil and was largely influenced by the oil phase as its normalized in the presence of oil is 0.8 times smaller than that in the absence of oil.
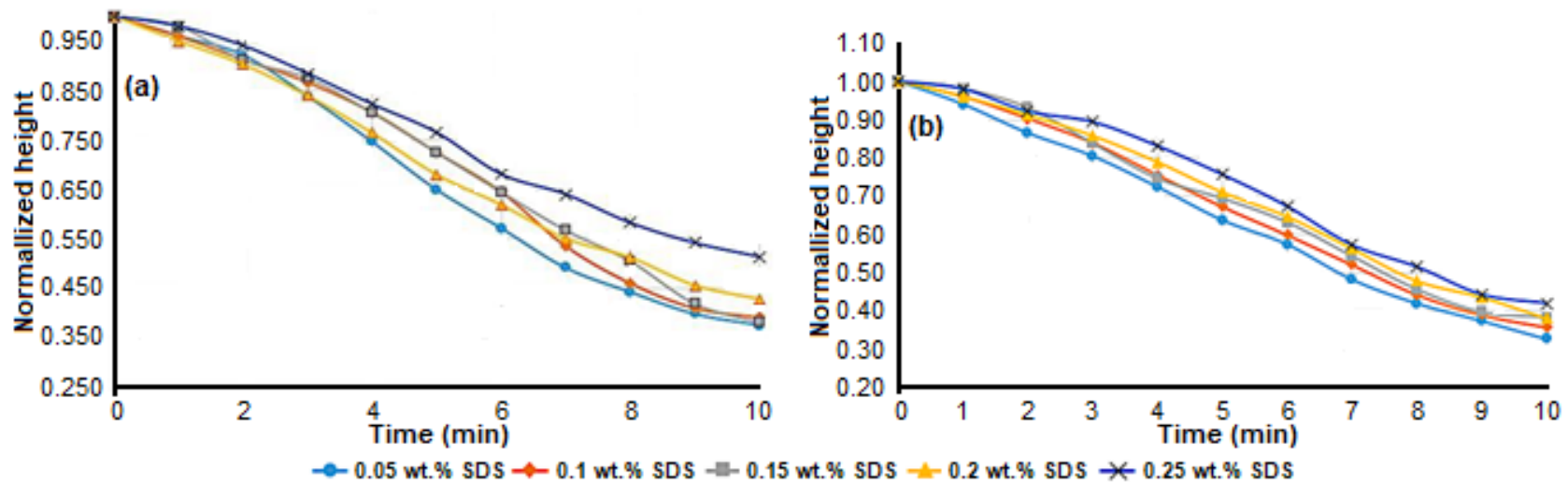

Fig 2. (a) Foam life for brine solution and mixture of $0.05,0.1,0.15,0.2$, and $0.25 \mathrm{wt}$.\% SDS surfactant in absence of white oil. (b) Foam life for brine solution and mixture of 0.05, 0.1, 0.15, 0.2 and $0.25 \mathrm{wt}$.\% SDS surfactant in presence of white oil
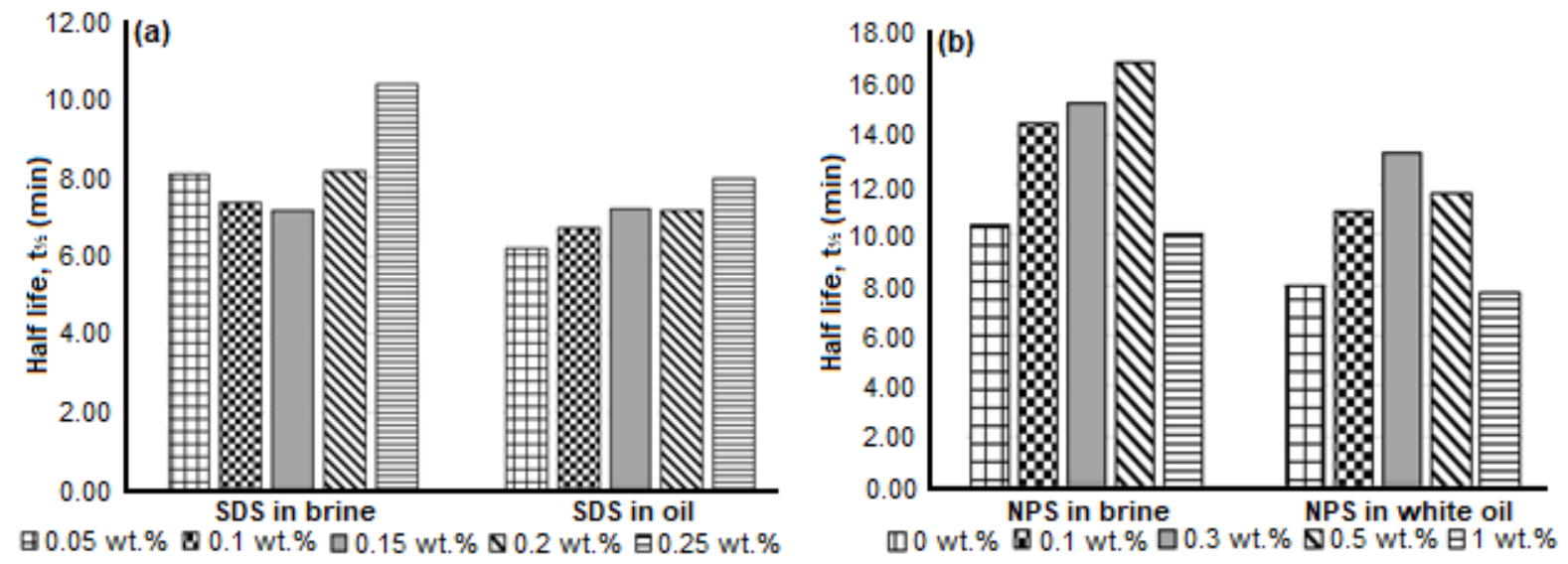

Fig 3. Effect of SDS surfactant and $\mathrm{SiO}_{2}$ nanoparticles concentration on foam stability in term of half-life. (a) Comparison of different wt.\% of SDS in brine and white oil (b) Comparison of different wt.\% of nanoparticles in brine and white oil 


\section{Effect of SDS Surfactant and $\mathrm{SiO}_{2}$ Nanoparticle Concentration on Foam Stability}

The foam half-lives for the two cases of sodium dodecyl sulfate (SDS) surfactant are shown in Fig. 3(a). The longest foam half-lives were reported at $0.25 \mathrm{wt} . \%$ SDS for both absence and presence of white mineral oil, 10.39 and $7.98 \mathrm{~min}$, respectively. In other word, at 0.25 wt. $\%$ of SDS; the foam is the most stable with the lowest decay rate for foam height. The results of the SDS surfactant also emphasize the importance of surfactant concentration. As shown in Fig. 3(b), the highest foam half-lives with this $0.25 \mathrm{wt} \%$ SDS surfactant were achieved at $0.5 \mathrm{wt} . \%$ nanoparticles in the absence of white mineral oil and $0.3 \mathrm{wt} . \%$ nanoparticles with the presence of white mineral oil, which are 16.9 and $13.3 \mathrm{~min}$, respectively. The trend of graph for varying nanoparticles concentration showed a similar pattern as the half-life increases as concentration increases and dropped after a certain concentration preference of nanoparticles. However, the shortest foam half-life was reported with $1.0 \mathrm{wt} . \%$ of nanoparticles for both cases, 10.0 and $7.7 \mathrm{~min}$ respectively, which is worse than using surfactant alone. It is not recommended to use high concentrations of nanoparticles to achieve the best stability. These results suggest that the addition of nanoparticles, at certain concentrations, might help to slow the drainage of thin aqueous film and, therefore, to produce a more stable foam.

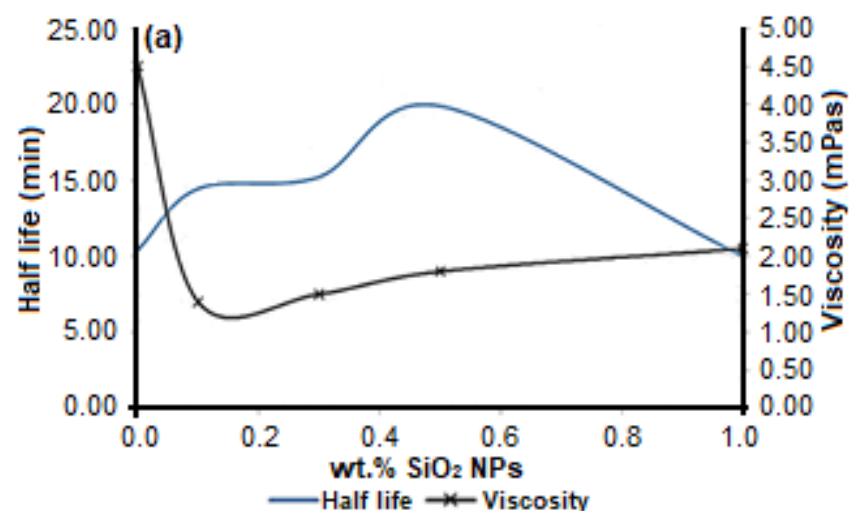

\section{Viscosity Test}

To further study the surfactant-nanoparticle mixtures, viscosity was determined, and the results are shown in Fig. 4. As seen in Fig. 4, the viscosity of nanoparticle-surfactant in brine solution showed a drastic drop for the concentration from 0 to $0.1 \mathrm{wt} . \%$ then increased steadily as the $\mathrm{SiO}_{2}$ concentration increases, but it is well below $2.5 \mathrm{mPa}$.s, while the half-life fluctuated with the increase of $\mathrm{SiO}_{2}$ concentration. This indicates that the higher nanoparticle concentration accrues in a more viscous liquid and the outlet rate of bulk foam can be delayed more in term of time that revamps the foam stability. In spite of that, the nanoparticle can partly reduce surfactant the surface adsorption, and this effect escalates with increasing nanoparticle concentration, hence resulted in the downturn of foaming capability of the nanoparticle enhanced foam. The former behavior is due to the fact that the aggregates formed move away from each other and a drastic decrease in viscosity is observed for an increase of $0 \mathrm{wt} . \%$ of the nanoparticle to $0.1 \mathrm{wt} . \%$ of nanoparticle. As the concentration increases, viscosity also increases because of increased nanoparticlesurfactant-water interactions, and hence, more nanoparticle and surfactant penetrates into the aqueous phase, increasing the micelle volume and resulting in increased viscosity. Thereupon, the stability of foam is strikingly enhanced by flourishing the viscosity.

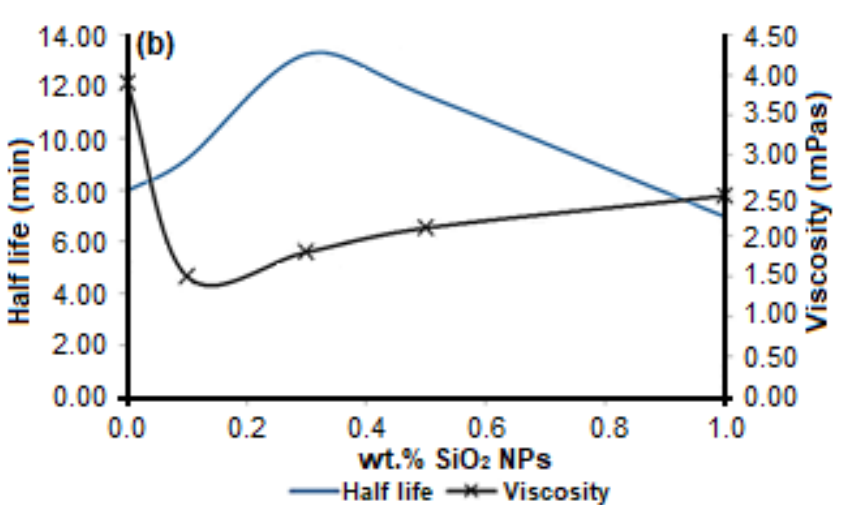

Fig 4. Viscosity and half-life for nanoparticle-surfactant in brine mixture (a) $\mathrm{SiO}_{2}$ in 0.25 wt.\% SDS and brine solution mixture of $0.0,0.1,0.3,0.5$, and $1.0 \mathrm{wt} \% \mathrm{SiO}_{2}$ in absence of oil. (b) $\mathrm{SiO}_{2}$ in $0.25 \mathrm{wt}$ \% SDS and brine solution mixture of $0.0,0.1,0.3,0.5$, and $1.0 \mathrm{wt} . \% \mathrm{SiO}_{2}$ in presence of oil 


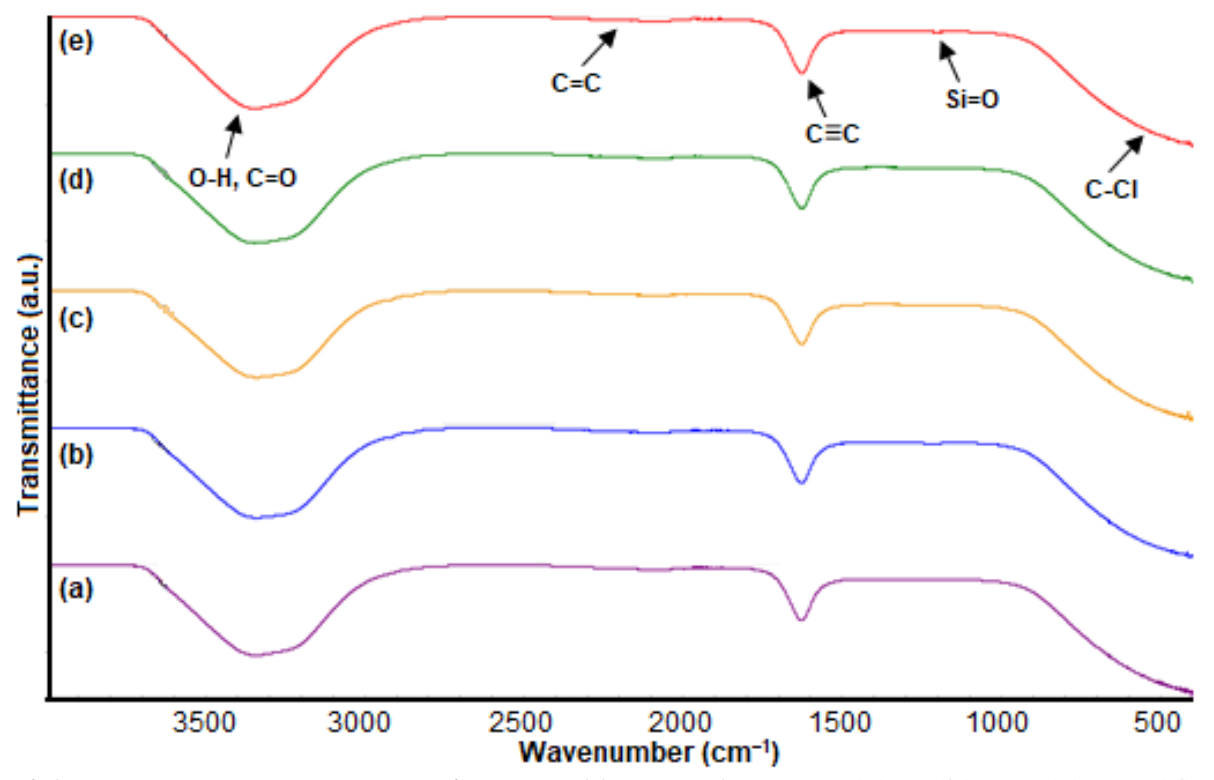

Fig 5. FTIR spectra of the $\mathrm{SiO}_{2} \mathrm{Nps}$ in $0.25 \mathrm{wt} . \%$ of SDS and brine solution at (a) 0, (b) 0.1, (c) 0.3 , (d) 0.5 , and (e) 1.0 wt.\%

\section{Characterization of the Synthesized $\mathrm{SiO}_{2}$ Nanoparticles Solution}

The FTIR measurements of the nanofluid with surfactant in brine solution were carried out to identify the possible interactions between nanoparticles and bioactive molecules, which may be responsible for the synthesis and stabilization of nanoparticles with the foamer agent used. The mechanism of surfactant adsorption on particle surfaces takes place into several different manners which are due to; chemical bonding, hydrogen bonding, hydrophobic bonding and governed by the initial interaction between silica surface van der Waals forces [17]. As being defined by Condon [18], physisorption or generally means physical adsorption takes place due to the attractions between solute molecules and adsorbent which did not involve any chemical interaction except the relatively weak forces encountered at the surface of the solid by means the hydrogen bonding and van der Waals forces.

The FTIR spectra of the nanofluid mixture at different concentration were recorded in the wavenumber range of $400-4000 \mathrm{~cm}^{-1}$. Fig. 5 presents the spectra of the $\mathrm{SiO}_{2}$ nanoparticle mixture in 0.25 wt.\% SDS and brine solution before gas sparging in which organic bonding can be detected in the range of $400-4000 \mathrm{~cm}^{-1}$. With adding nanoparticle into the surfactant and brine suspension, these bonding vibrations gradually increase with more spikes even if these peaks are small in consideration to the alcohol bond, $\mathrm{O}-\mathrm{H}$, and alkyne bond, $\mathrm{C} \equiv \mathrm{C}$. As the concentration of nanoparticle is increased above $0.05 \mathrm{wt} . \%$, these vibrations difference become virtually undetectable. The amide linkages, $\mathrm{C}=\mathrm{O}$ between nanoparticle in SDS and brine suspension give rise to well-known signatures in the infrared region of the electromagnetic spectrum. The FTIR spectrum reveals a significantly broad band at an approximate range from 3600 and $3000 \mathrm{~cm}^{-1}$ that corresponds to the bending vibrations of the amide bands of the nanoparticle.

In the approximate range from 1500 to $500 \mathrm{~cm}^{-1}$ and 3500 to $3000 \mathrm{~cm}^{-1}, \mathrm{C}=\mathrm{O}$ and $\mathrm{Si}=\mathrm{O}$ bending and stretching vibrations due to the polyethylene oxide (PEO) of surfactant SDS and $\mathrm{SiO}_{2}$ can be observed. These bands show increase intensities with increasing concentration from 0 to $0.1 \mathrm{wt} \%$. The $\mathrm{O}-\mathrm{H}$ stretching vibration that gives rise to absorption from 3500 to $3200 \mathrm{~cm}^{-1}$ can be tentatively associated with adsorbed water in a brine solution. Strong absorptions at 1085 and $800 \mathrm{~cm}^{-1}$ also can be observed. These absorptions identified respectively as the $\mathrm{Si}-\mathrm{O}-\mathrm{Si}$ asymmetry and symmetry bands. Theoretically, silica surfaces are inherently presence with silanol groups, at which under 
an adequate concentration on the surface, gives the silica hydrophilic properties and experimentally shown, the density of silanol highly influence on the adsorption behavior of solute species. Upon interaction with adsorbates, the $\mathrm{OH}$ groups exist particularly act as centers of molecular adsorption of competently forming a hydrogen bond [19].

\section{- CONCLUSION}

Foam stability and characteristics were studied using varies surfactants and nanoparticle concentrations in the absence and presence of white mineral oil. Foam stability enhances as surfactants concentrations increases. High surfactant concentration solutions were characterized by fine and fairy uniform distributed bubble size whereas lower concentrations clearly reveal a different foam texture. The addition of nanoparticles at certain concentration to this specific synthetic saltwater and oil shows promise when it comes to increasing the half time of foam as it might help to slow the drainage of thin aqueous film and, therefore, to produce a more stable foam. The $0.5 \mathrm{wt} . \% \mathrm{SiO}_{2}$ nanoparticles enhanced foam formed the most lasting in the absence of white mineral oil as its $t_{1 / 2}$ in the presence of oil is 0.6 times smaller than that in the absence of oil. Overall, the results with added nanoparticles are better than the results with no added nanoparticles in term of foam stability. Thus, nanotechnology has the capability to have a constructive fallout on the EOR process. It is recommended to conduct further research to fully improve the stability of nanostabilized foam in tertiary petroleum production by: 1) Study on the stability through porous media by means of dynamic foam testing; 2) Various temperature condition for stability testing can be studied further; and 3) Conduct study on another type of nanoparticles, surfactants and oil.

\section{- ACKNOWLEDGMENTS}

Thanks to the Faculty of Chemical and Natural Resources Engineering Universiti Malaysia Pahang and Research \& Innovation center for giving the research grant RDU180325 to run out the research study under an internal research project scheme.

\section{- REFERENCES}

[1] Liu, Q., Zhang, S., Sun, D., and Xu, J., 2010, Foams stabilized by Laponite nanoparticles and alkylammonium bromides with different alkyl chain lengths, Colloids Surf., A, 355 (1-3), 151-157.

[2] Schramm, L.L., and Wassmuth, F., 1994, Foams: Basic principles, Adv. Chem., 242, 3-45.

[3] Gonzenbach, U.T., Studart, A.R., Tervoort, E., and Gauckler, L.J., 2006, Stabilization of foams with inorganic colloidal particles, Langmuir, 22 (26), 10983-10988.

[4] Grigg, R.B., and Mikhalin, A.A., 2007, Effects of Flow Conditions and Surfactant Availability on Adsorption, International Symposium on Oilfield Chemistry, Society of Petroleum Engineers, 28 February-2 March 2007, Houston, Texas, U.S.A., $1-7$.

[5] Isari, A.A., Moradi, S., Bachari, Z., and Mahmoodi, H., 2017, Application of natural surfactants for enhanced oil recovery-Critical review, The $4^{\text {th }}$ International Conference On Oil, Gas and Petrochemical, Tehran University, 8-9 May 2017, Tehran, Iran.

[6] Horozov, T.S., 2008, Foams and foam films stabilised by solid particles, Curr. Opin. Colloid Interface Sci., 13 (3), 134-140.

[7] Bai, T., Liu, Y., Liu, J., Yu, C., Jiang, W., and Fan Y., 2019, A comparison of different surfactants on foam stability in foam sclerotherapy in vitro, $J$. Vasc. Surg., 69 (2), 581-591.

[8] Wang, C., and Li, H.A., 2016, Stability and mobility of foam generated by gas-solvent/surfactant mixtures under reservoir conditions, J. Nat. Gas Sci. Eng., 34, 366-375.

[9] Tang, F.Q., Xiao, Z., Tang, J.A., and Jiang, L., 1989, The effect of $\mathrm{SiO}_{2}$ particles upon stabilization of foam, J. Colloid Interface Sci., 131 (2), 498-502.

[10] Murray, B.S., and Ettelaie, R., 2004, Foam stability: Proteins and nanoparticles, Curr. Opin. Colloid Interface Sci., 9 (5), 314-320.

[11] Agista, M.N., Guo, K., and Yu, Z., 2018, A state-of- 
the-art review of nanoparticles application in petroleum with a focus on enhanced oil recovery, Appl. Sci., 8 (6), 871.

[12] Hirasaki, G.J., Miller, C.A., and Puerto, M., 2008, Recent Advances in Surfactant EOR, SPE Annual Technical Conference and Exhibition, Society of Petroleum Engineers, 21-24 September, Denver, Colorado, USA.

[13] ASTM Standard D6082-12, 2012, Standard Test Method for High Temperature Foaming Characteristics of Lubricating Oils, ASTM International, West Conshohocken, PA.

[14] Nishioka, G., and Ross, S., 1981, A new method and apparatus for measuring foam stability, J. Colloid Interface Sci., 81 (1), 1-7.

[15] Manan, M.A., Farad, S., Piroozian, A., and Esmail, M.J.A., 2015, Effects of nanoparticle types on carbon dioxide foam flooding in enhanced oil recovery, $J$. Pet. Sci. Technol., 33 (12), 1286-1294.

[16] Eren, T., 2004, Foam Characterization: Bubble Size and Texture Effects, Thesis, Middle East Technical University.

[17] Parida, S.K., Dash, S., Patel, S., and Mishra, B.K., 2006, Adsorption of organic molecules on silica surface, Adv. Colloid Interface Sci., 121 (1-3), 77-110.

[18] Condon, J.B., 2006, "An Overview of Physisorption" in Surface Area and Porosity Determinations by Physisorption: Measurements and Theory, $1^{\text {st }}$ Ed., Elsevier Science, Amsterdam, 1-27.

[19] Horozov, T.S., Binks, B.P., Aveyard, R., and Clint, J.H., 2006, Effect of particle hydrophobicity on the formation and collapse of fumed silica particle monolayers at the oil-water interface, Colloids Surf., A, 282-283, 377-386. 